phospholipid fatty acid patterns were measured at baseline and at the end of the study. Children with ADHD had low levels of plasma DHA at baseline. Differences between DHA and placebo groups, determined by analysis of variance, showed that plasma phospholipid DHA content of the supplemented group was 2.6-fold higher at the end of the study compared to the placebo group. Measures of inattention and impulsivity and parental questionnaires showed no significant improvement in the DHA group. Errors of omission on the TOVA test increased significantly in the DHA group but not in the placebo group, and errors of commission decreased significantly in the placebo group but not in the DHA group. (Voigt RG, Llorente AM, Jensen CL et al. A randomized, double-blind, placebo-controlled trial of docosahexaenoic acid supplementation in children with attention-deficit/hyperactivity disorder. $\perp$ Pediatr August 2001;139:189-196). (Reprints: Robert G Voigt MD, Division of Developmental and Behavioral Pediatrics, Department of Pediatrics and Adolescent Medicine, Mayo Clinic, 200 First Street SW, Baldwin $3 \mathrm{~A}$, Rochester, MN 55905).

COMMENT. A 4-month trial of DHA supplement, administered while continuing effective stimulant medication, had no measurable significant effect on ADHD symptoms observed by the parents. Similarly, objective tests of attentiveness and impulsivity, administered after a 24 hour withdrawal of medication, showed no significant benefit in children receiving DHA supplement. It was assumed that any lasting or withdrawal effect of stimulant medication had dissipated in the short 24 hour drug holiday. The well known "rebound" phenomenon when MPH is discontinued could have vitiated any possible benefit from DHA. (Millichap JG. Attention Deficit Hyperactivity and Learning Disorders. PNB Publ, 2001; Schachar RJ et al, 1997).

The limitations of this study include the following: 1) trial subjects who were already benefited by stimulant medication; 2) the use of a single dose of DHA supplement; 3 ) failure to include arachidonic acid and other fatty acids that are often at low levels in children with ADHD. Future controlled studies might be conducted in cases of ADHD previously untreated with stimulant medication, using more than one dose of supplement, and with multiple fatty acids.

Kemper KJ, in an editorial (L Pediatr August 2001;139:173-174), advocates the testing of further dietary supplements, and greater assurances of safety, purity, and potency before marketing.

For a review of dietary supplements in ADHD, see Progress in Pediatric Neurology III. PNB Publ, 1997;pp209-210. Mitchell EA et al, in 1987, found that DHA and arachidonic acid serum levels were significantly lower in 44 hyperactive children compared to 45 age- and sex-matched controls. Stordy BJ, in 1995, reported that DHA supplements improved dark adaptation (scotopic vision) in 5 adults with dyslexia. She later proposed DHA as a treatment for ADD and short term memory problems.

\title{
CLONIDINE IN HYPERACTIVE, MENTALLY RETARDED CHILDREN
}

The effects of oral clonidine on hyperactive children with comorbid mental retardation were examined in 10 children (mean age 7.6 years) treated at the Department of Psychiatry, King George's Medical College, Lucknow, India. In a 12-week, double-blind, randomized, placebo-controlled trial, using 3 fixed doses (4, 6 , and $8 \mathrm{mcg} / \mathrm{kg} /$ day), Parent Questionnaire and Clinician Rating Scales showed a dose-related effect on hyperactivity, inattention, and impulsivity, and improvements in conduct abnormalities, frustration tolerance, cooperation, and interest in tasks. Whereas hyperactivity and impulsivity were improved with increasing doses from 4- to 8 -mcg, improved attention occurred only at the 4- and 
6-mcg doses. Drowsiness occurred in 5 children, and 1 child developed dry mouth and anorexia Systolic blood pressure was lowered $3 \%$ with $4-\mathrm{mcg}$ dose, $6.9 \%$ with 6-mcg, and $8.9 \%$ with 8 -mcg doses. Clonidine was considered safe and effective in hyperkinetic children with mental retardation. (Agarwal V, Sitholey P, Kumar S, Prasad M. Double-blind, placebo-controlled trial of clonidine in hyperactive children with mental retardation. Mental Retardation August 2001;39:259-267). (Reprints: Dr Vivek Agarwal, B-1, 10/69 sector K-Aliganj, Lucknow-226024, India).

COMMENT. In this rather limited cohort of patients, the efficacy and safety of clonidine in the management of hyperactive, mentally retarded children has been demonstrated by controlled trial. Clonidine is a second line treatment for ADHD and is indicated primarily in patients with comorbid tics, oppositional defiance disorder, and sleep problems.

Hypoglycemic effect of clonidine. Four cases of hypoglycemia associated with clonidine stimulation of growth hormone secretion are reported from the Hospital for Sick Children, Toronto, Ontario (Huang $\mathrm{C}$, Banerjee $\mathrm{K}$, Sochett E et al. LPediatr August 2001;139:323-324). Only one patient had growth hormone deficiency. Drowsiness after $0.15 \mathrm{mg} / \mathrm{m}^{2}$ dose AM may prolong fasting and mask early signs and symptoms, leading to severe hypoglycemia. One patient aged 3 years had a generalized tonic-clonic seizure with a serum glucose of 2.3 $\mathrm{mmol} / \mathrm{L}$ EEG and head CT scan were normal.

Hypoglycemia is not a reported adverse reaction to clonidine in ADHD children, but blood sugar levels should be monitored in patients with suggestive symptoms. The PDR lists a transient elevation of blood glucose, not hypoglycemia, as a rare adverse reaction of clonidine.

\section{VASCULAR DISORDERS}

\section{PRE- OR PERINATAL ARTERIAL ISCHEMIC STROKE}

Risk factors, coagulation profiles, and outcomes in 22 children with presumed perinatal infarct were studied at The Hospital for Sick Children, Toronto, and Chedoke McMaster Hospital, Hamilton, both participants in the Canadian Pediatric Ischemic Stroke Registry. Criteria for inclusion were the following: 1) normal neonatal neurological history, 2) hemiparesis and/or seizures first recognized after two months of age, and 3) CT or MRI showing remote cerebral infarct. Median age at presentation was 6 months. Median age at last visit was 4 years (range, 8 months to 16.5 years). Eighteen of 22 mothers had gestational or obstetrical risk factors, including preeclampsia (4), maternal infection (4), gestational diabetes (3), premature delivery, bleeding, and breech presentation. Fourteen children had coagulation abnormalities including anticardiolipin antibody (ACLA) in 12, and activated protein C resistance (APCR) in 3. Six had a family history of thrombosis. Echocardiograms were normal. Hemiparesis, right sided in 14, persisted in all patients, 12 had speech, behavior, or learning problems, and 5 had persistent seizures. Stroke did not recur, despite persistence of ACLA or other clotting abnormalities. (Golomb MR, MacGregor DL, Domi $\mathrm{T}$ et al. Presumed pre- or perinatal arterial ischemic stroke: risk factors and outcomes. Ann Neurol August 2001;50:163-168). (Respond: Dr Gabrielle A deVeber, Division of Neurology, The Hospital for Sick Children, 555 University Ave, Toronto M5G 1X8, ON, Canada).

COMMENT. ACLA is a risk factor for ischemic stroke in the fetus and neonate, and recognition of hemiparesis may be delayed to age 6 months or later. 